\title{
Higher risk of late-onset sepsis in very low birth weight male preterm infants
}

\author{
David Ramiro-Cortijo', Carlos Zozaya-Nieto², Ángel L. López de Pabloํ․ M. Rosario López-Giménez \\ Miguel Saenz de Pipaon ${ }^{2}$ and Silvia M. Arribas ${ }^{1 *}$
}

${ }^{1}$ Department of Physiology, School of Medicine, Autonomous University of Madrid; ${ }^{2}$ Department of Pediatrics, Neonatology Services, La Paz University Hospital; ${ }^{3}$ Department of Preventive Medicine, Public Health and Microbiology, School of Medicine, Autonomous University of Madrid. Madrid, Spain

\begin{abstract}
Objetives: To evaluate male gender as a risk factor for perinatal comorbidities and growth in a population of very low birth weight (VLBW) premature infants. Materials and Methods: A population of 1390 VLBW premature infants admitted to Hospital La Paz (Spain) between 2005 and 2015 was retrospectively studied. We evaluated mortality rates, severity at admission, and incidence of the most prevalent comorbidities in prematurity and growth. Multivariate regression analysis was applied to evaluate the effect of gender on the variables studied. Results: No differences in gestational age, Apgar and clinical risk index for babies scores, and mortality rates were detected between the genders. Male infants showed a higher prevalence of the majority of the comorbidities of prematurity, bronchopulmonary dysplasia (BPD), and late-onset sepsis (LOS) being significant compared with females. Conclusions: Male infants had larger anthropometric parameters at birth without differences in body growth compared to females. Male gender is a risk factor for LOS and BPD in VLBW infants without influence on perinatal growth.
\end{abstract}

Key words: Male risk factor. Very low birth weight neonates. Late-onset sepsis.

\section{Introduction}

According to the World Health Organization, more than 15 million babies are born prematurely every year ${ }^{1}$. Preterm birth is associated with an increased risk of mortality, being the leading cause of death of children under 5 years. Prematurity is also associated with worse neurodevelopment, leading to cognitive, motor and behavioral impairment later in life, and with high morbidity, and particularly during admission. The most prevalent complications of preterm neonates are respiratory distress syndrome (RDS), bronchopulmonary dysplasia (BPD) ${ }^{2}$, necrotizing enterocolitis (NEC), germinal matrix hemorrhage $(\mathrm{GMH})$, intraventricular hemorrhage (IVH), periventricular hemorrhagic infarction (PVHI), patent ductus arteriosus (PDA), retinopathy of prematurity (ROP), and gut dysbiosis. Furthermore, due to skin immaturity and the need for invasive devices, there is a high risk of late-onset sepsis $(\text { LOS })^{3}$, which is associated with mortality and worse neurodevelopment.

Preterm birth is a growing problem worldwide, both in low- and high-income countries. Therefore, given the important consequences for the future health of the individual, gaining insight into the reasons for this rise is essential. However, the etiology of prematurity is complex due to its multifactorial origin, including both

\section{Correspondence:}

Silvia M. Arribas

E-mail: silvia.arribas@uam.es
Available online: 30-11-2018

Date of reception: 22-06-2018

Date of acceptance: 25-08-2018

DOI: 10.24875/RMU.M18000021

\section{Medicina Universitaria. 2018;20(3):118-124} www.medicinauniversitaria.org

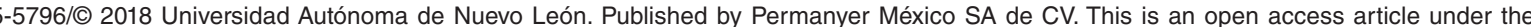
CC BY-NC-ND license (http://creativecommons.org/licenses/by-nc-nd/4.0/). 
biological and social factors, which are usually interrelated. In low- and middle-income societies, situations of poverty or gender inequity leading to malnutrition, poor health care or maternal infections during pregnancy $^{4}$ are important causes of prematurity ${ }^{1,5}$. In high-income countries, the risk factors are different. The rise in the age of first pregnancies is an important contributor; it is directly related to pregnancy complications, which are an important cause of prematurity. The increase in maternity age is also linked to a decline in fertility, associated with the use of assisted reproduction techniques and multiple pregnancies, another biological factor associated with preterm birth.

In addition to the factors mentioned above associated with prematurity, some studies suggest that male gender could be an additional risk factor. In this sense, there is evidence of a higher prematurity rate among male neonates compared to females. This fact has been reported in populations of different racial and socioeconomic backgrounds and is more evident in extreme preterm infants ${ }^{6-9}$. Some studies also show that male extreme preterm infants have a higher risk of mortality before first hospital discharge than female extreme preterm infants ${ }^{10}$. However, gender is usually taken into consideration in epidemiological studies of premature babies, few of them evaluate the influence of gender in the development of comorbidities as main outcome.

We hypothesize that male preterm infants would be at higher risk of comorbidities and mortality and have a worse growth rate during the first neonatal admission, compared to females. Therefore, the aims of the present study are to evaluate, in a population of very low birth weight (VLBW) premature infants, possible differences between males and females in: (1) mortality rate, (2) severity at admission, (3) incidence of the most common comorbidities associated with preterm birth, and (4) growth rates (body weight, length, and head circumference) during admission in the neonatal intensive care unit (NICU).

\section{Materials and methods}

\section{Study population}

This is a retrospective study of prospectively collected data of a population of preterm infants admitted to a single NICU at La Paz University Hospital (Madrid, Spain), from January 2005 to December 2015. The inclusion criteria were VLBW infants (VLBW, birth weight $<1500 \mathrm{~g}$ ), born between $>24$ and $\leq 32$ weeks.
The study was approved by the La Paz University Hospital Research Ethics Committee.

\section{Basic clinical parameters}

\section{Definitions}

Extreme premature infants are those born at $<28$ weeks of gestation, and very preterm infants are those born between $\geq 28$ and $\leq 32$ weeks. Complete antenatal corticosteroids therapy consists of an administration of 2 doses of intramuscular betamethasone $24 \mathrm{~h}$ apart. Advanced resuscitation at birth was considered when endotracheal intubation, heart massage or adrenalin administration was needed. To quantify severity at admission, clinical risk index for babies (CRIB) ${ }^{1}$ and Apgar scores, as well as the need for advanced resuscitation, were evaluated. A low Apgar score was defined as values $<7$ measured at $5 \mathrm{~min}$.

Borderline personality disorder (BDP) was defined as needing oxygen for at least 28 days, moderate-severe BDP being only those cases which required supplementary oxygen at 36 weeks postmenstrual age $(\mathrm{PMA})^{2}$. NEC was considered at only Stage IIA and above, according to a modified Bell's criteria ${ }^{3}$. GMH and IVH, according to Volpe's classification. LOS was defined as clinical signs of septicemia along with a positive blood culture after $72 \mathrm{~h}$. PDA was considered as the presence of hemodynamic alterations, based on echocardiographic and clinical criteria. ROP was classified according to international classification ROP5. Mortality was considered as exitus during the first neonatal admission.

During admission, infant growth was evaluated by (1) weight gain from birth to 36 weeks PMA, assessed as increase in $\mathrm{g} / \mathrm{kg} / \mathrm{day}$, according to Patel et al. ${ }^{17,18}$, (2) body length and head circumference increase from birth to discharge, evaluated as increase in $\mathrm{cm} /$ week, (3) weight z-scores at birth and at 36 weeks PMA, using Fenton growth curves, and (4) extrauterine growth restriction (EUGR) in infants born adequate or long for gestational age, defined as a decrease in standard deviation score between weight z-score at birth and at 36 weeks PMA > 0.67, according to Ong et al. ${ }^{11}$.

\section{Statistical analyses}

The study was originally designed to evaluate the influence of gender on clinical outcomes and growth. Descriptive statistics are expressed as a mean \pm standard 
Table 1. Epidemiological and clinical features of the population.

\begin{tabular}{|l|c|c|c|c|}
\hline Gestational Characteristics & VLBW infants $(\mathbf{n}=\mathbf{1 3 9 0})$ & Male $(\mathbf{n}=\mathbf{7 2 5})$ & Female (n= 665) & $\mathbf{p}$-value \\
\hline Gestational age, weeks & $28.2 \pm 2.2$ & $27.7 \pm 2.2$ & $27.9 \pm 2.2$ & 0.142 \\
\hline Gestational age, days & $197.5 \pm 15.5$ & $194.4 \pm 15.7$ & $195.6 \pm 15.3$ & 0.142 \\
\hline Birth weight, g & $1025.8 \pm 268.3$ & $1044.6 \pm 268.5^{*}$ & $1005.2 \pm 266.8$ & 0.006 \\
\hline Small for gestational age (\%) & $178(12.8)$ & $101(13.9)$ & $77(11.6)$ & 0.126 \\
\hline Mode of delivery, cesarean section (\%) & $1043(75)$ & $528(72.8)^{*}$ & $515(77.4)$ & 0.047 \\
\hline Antenatal corticosteroid therapy (\%) & $1015(74)$ & $509(71.3)^{*}$ & $506(77.1)$ & 0.014 \\
\hline
\end{tabular}

Data are expressed as mean \pm standard deviation or as frequency and percentage. Student's $t$-test in continuous variables or Chi-squared in qualitative variables. ${ }^{*} p<0.05$ comparing male to female.

VLBW: very low birth weight

deviation for continuous variables or $n(\%)$ for categorical variables.

To quantify the association between gender and the clinical variables, univariate analysis was applied. Student's $t$-test or two-tailed Chi-squared tests were used for continuous or categorical variables, respectively. A multivariate regression model, linear or logistic, was applied to evaluate the independent effect of gender on clinical and growth variables, weight at birth, and gestational age being the potential confounding covariates. $p<0.05$ was considered statistically significant. Statistical analysis was performed with Stata 2013 (statistical software, Stata Corp. Texas, USA).

\section{Results}

The number of VLBW infants who were admitted to the NICU of La Paz University Hospital from January 2005 to December 2015 was 1696, with 1390 being eligible according to the inclusion criteria. The clinical characteristics of the population are summarized in table 1.

In the studied population of VLBW infants, we did not detect statistical differences in the percentage of males and females according to gestational age neither in extreme premature nor in very preterm infants (Fig. 1).

We did not detect statistical differences in the percentage of male and female infants with an Apgar score $<7$ or in the CRIB score values between genders. However, we found a significantly higher percentage of males that required advanced resuscitation at birth compared to females (Table 2). This difference was not statistically significant when a multivariate analysis was used, adjusting for gestational age and birth weight (odds

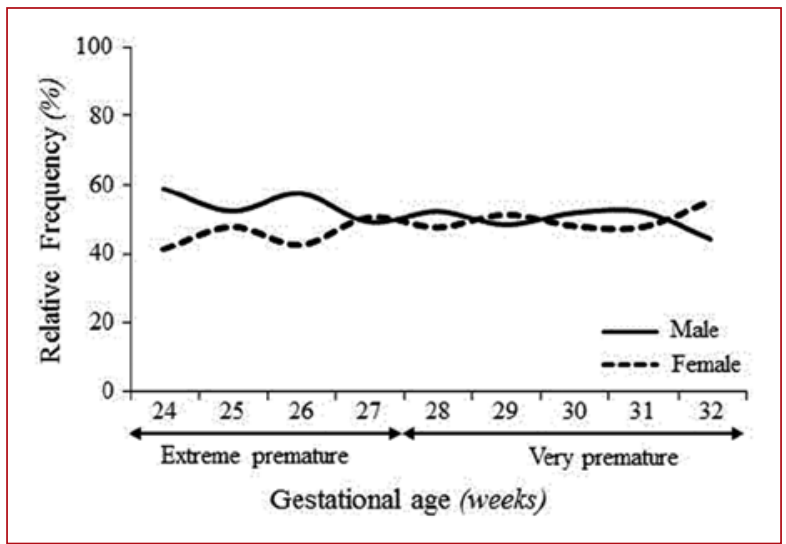

Figure 1. Relative frequencies of births at different gestational ages according to sex in a population of very low birth weight premature infants ( $n=1390)$.

ratio $[\mathrm{OR}] 1.2 ; 95 \%$ confidence interval [Cl] 0.9-1.6; $p=0.095)$ and the need for advanced resuscitation was only influenced by gestational age (OR $0.57 ; 95 \%$ Cl 0.52-0.63; $p=0.0001$ ).

\section{Morbimortality}

On comparing male and female infants, no differences were found in the incidence of RDS, PDA, NEC, ROP, GMH/IVH, and PVHI. However, a higher incidence of LOS and BPD were found in male infants (Table 2). Multivariate analysis, adjusting by gestational age and birth weight, demonstrated that male gender is a risk factor for LOS (OR 1.3; 95\% Cl 1.03-1.7) and BPD (OR 1.7; 95\% Cl 1.2-2.4). No significant differences in the proportion of mortality according to gender (males $13.9 \%$; females $11.9 \% ; p=0.284$ ) were found. 
Table 2. Apgar and CRIB scores at birth and relative frequencies of relevant comorbidities associated with prematurity according to sex in a population of very low birth weight premature infants.

\begin{tabular}{|l|c|c|c|c|}
\hline Neonatal Scores and Comorbidities & VLBW infants $(\mathbf{n}=\mathbf{1 3 9 0})$ & Male $(\mathbf{n}=\mathbf{7 2 5})$ & Female (n= 665) & $p$-value \\
\hline Apgar score $<7$ at 5 min (\%) & $249(18)$ & $141(19.4)$ & $109(16.4)$ & 0.150 \\
\hline CRIB score & $3.2 \pm 3.5$ & $3.3 \pm 3.4$ & $3.3 \pm 3.5$ & 0.985 \\
\hline Advanced resuscitation at birth (\%) & $533(38.3)$ & $296(40.8)^{*}$ & $237(35.6)$ & 0.047 \\
\hline Respiratory distress syndrome (\%) & $855(61.5)$ & $460(63.4)$ & $395(59.4)$ & 0.120 \\
\hline Bronchopulmonary dysplasia (\%) & $607(49.6)$ & $334(52.9)^{*}$ & $273(46)$ & 0.020 \\
\hline Necrotizing enterocolitis (\%) & $80(5.8)$ & $45(6.2)$ & $35(5.3)$ & 0.720 \\
\hline Patent ductus arteriosus (\%) & $620(44.7)$ & $326(45)$ & $294(44.3)$ & 0.832 \\
\hline Germinal matrix hemorrhage (\%) & $404(31.2)$ & $460(63.5)$ & $395(59.4)$ & 0.099 \\
\hline Late onset sepsis (\%) & $482(35.6)$ & $268(38.1)^{*}$ & $214(32.9)$ & 0.046 \\
\hline Retinopathy of prematurity (\%) & $327(28.4)$ & $173(28.8)$ & $154(27.9)$ & 0.720 \\
\hline
\end{tabular}

Data are expressed as mean \pm standard error or as frequency and percentage. Student's $t$-test in continuous variables or Chi-squared in qualitative variables. ${ }^{*} p<0.05$ comparing male to female.

VLBW: very low birth weight, CRIB: clinical risk index for babies

Table 3. Anthropometric parameters at birth and gain of weight, height, and cephalic perimeter from birth to discharge.

\begin{tabular}{|c|c|c|c|c|}
\hline Anthropometric Parameters & VLBW infants ( $n=1390$ ) & Male $(n=725)$ & Female $(n=665)$ & $p$-value \\
\hline Birth weight, $g$ & $1025.8 \pm 268.3$ & $1044.6 \pm 268.5^{*}$ & $1005.2 \pm 266.8$ & 0.006 \\
\hline z-score body weight & $-0.3 \pm 0.8$ & $-0.3 \pm 0.9$ & $-0.3 \pm 0.8$ & 0.857 \\
\hline Birth length, $\mathrm{cm}$ & $35.6 \pm 3.4$ & $35.7 \pm 3.5$ & $35.5 \pm 3.4$ & 0.317 \\
\hline Birth head circumference, $\mathrm{cm}$ & $25.4 \pm 2.4$ & $25.5 \pm 2.4^{*}$ & $25.2 \pm 2.5$ & 0.008 \\
\hline Body weight gain, $\mathrm{g} / \mathrm{kg} / \mathrm{day}$ & $12.5 \pm 2.6$ & $12.4 \pm 2.6$ & $12.6 \pm 2.6$ & 0.184 \\
\hline Body length growth, $\mathrm{cm} /$ weeks & $0.9 \pm 0.4$ & $0.8 \pm 0.4$ & $0.9 \pm 0.3$ & 0.547 \\
\hline Head circumferences growth, $\mathrm{cm} /$ weeks & $0.7 \pm 0.3$ & $0.7 \pm 0.3$ & $0.7 \pm 0.3$ & 0.281 \\
\hline Extrauterine growth restriction (\%) & $905(65)$ & $(58.3)^{*}$ & (49.5) & 0.001 \\
\hline
\end{tabular}

Data are expressed as mean \pm standard error or as frequency and percentage. Student's $t$-test in continuous variables or Chi-squared in qualitative variables. ${ }^{*} p<0.05$ comparing male to female. VLBW: very low birth weight

\section{Growth during admission}

Anthropometric parameters of the population at birth are shown in table 3. At birth, male infants exhibited a significantly higher mean body weight compared to females, the difference between genders being $39.4 \pm$ $14.4 \mathrm{~g}(95 \% \mathrm{Cl} 11.2-67.6 ; p=0.006)$. Mean head circumference was also significantly larger in male infants compared to females, the difference between genders being $0.35 \pm 0.13 \mathrm{~cm}(95 \% \mathrm{Cl} 0.09-0.6 ; p=0.008)$. However, no statistical differences between the genders in mean body length were detected.
We did not detect statistically significant differences between genders in body weight gain from birth to 36 weeks PMA. Similarly, no differences between genders were detected in body length and head circumference growth rate from birth to discharge.

Regarding EUGR among preterm infants born adequately or large for gestational age, a significantly higher proportion of males was found. However, after adjusting by gestational age, birth body weight, BPD, and LOS, we did not find a statistically significant difference in EUGR between male and female infants (OR $1.1 ; 95 \% \mathrm{Cl} 0.9-1.4 ; p=0.33$ ). 


\section{Discussion}

The incidence of prematurity has risen in the past two decades worldwide. Even though the survival rates of preterm babies have increased significantly, particularly in high-income countries, due to improved neonatal care, prematurity remains the main cause of perinatal mortality and morbidity, as well as long-term loss of human potential among survivors. In low-income countries, prematurity is directly associated with poor maternal nutrition and a high rate of maternal infections; therefore, improving health-care systems would likely reduce the rate. However, prematurity also remains high in middle- and high-income countries, despite major advances in pregnancy health care. Among other factors, in high-income countries, the gradual increase in the age of the first pregnancy, due to access to female employment, increases the risk of pregnancy complications and prematurity.

Furthermore, there is an increase of assisted reproductive techniques leading to multiple pregnancies that are also associated with prematurity. In addition to these social factors, the influence of gender on prematurity is gradually gaining attention ${ }^{9}$. However, this information is still insufficient, and more studies are needed to prove clear evidence and modify neonatal care protocols according to the gender of the individual. The present study was designed to add information to this growing area of research.

Several studies indicate that male infants are at higher risk of prematurity in comparison to females. A meta-analysis of several populations extracted from published references shows that, overall, there is a higher proportion of male babies delivered preterm ${ }^{10}$. More recent studies in populations of different racial and socioeconomic backgrounds ${ }^{12-16}$, including twin pregnancies ${ }^{17}$, confirm these data and reinforces the conclusion that the male gender is a risk factor for prematurity. These studies have also evidenced that this higher proportion of male preterm births is particularly evident at low gestational ages. We also observed a tendency toward a higher proportion of males in low gestational ages, but no statistical difference was found. This may be due to the characteristics of the population and limitations in the sample size. The above-mentioned studies were performed on very large regional or national cohorts, including all ranges of birth weight, while the present work was limited to one single NICU and was restricted to VLBW infants.

Regarding the relationship between gender and severity at admission, some studies have reported lower
Apgar score values in premature male infants, in relation to females ${ }^{10,13}$. In a population of VLBW infants, Stevenson and co. also found a higher percentage of males with lower Apgar scores and a need for physical and pharmacological assistance, which was attributed to lower exposure to antenatal steroids in male infants ${ }^{18}$. In our population of VLBW infants, no differences according to gender in Apgar or CRIB scores were detected, but a larger proportion of male infants required resuscitation, suggesting a higher severity at admission. However, when adjusted by gestational age and birth weight, this difference was not statistically significant, suggesting that these factors have a higher contribution than gender. In our study, the observed differences in resuscitation needed are not likely attributed to differences in corticosteroid treatment, which was homogeneous in the population.

Many studies have focused on the influence of gender on the incidence of prematurity, and fewer have evaluated the effect on comorbidities and specifically evaluated all the main pathologies in prematurity with multivariate analysis. In fact, the influence of gender on neonatal outcome has not been thoroughly evaluated, since gender has usually been considered as a confounding factor. Peelen et al., in a large cohort of premature infants in the Netherlands between 1999 and 2010 , reported that males are at increased risk of neonatal morbidity, evaluated as the presence of any of the most common pathologies of prematurity or Apgar score $<7$ at $5 \mathrm{~min}^{16}$. In our population of VLBW infants, the individual evaluation of the different comorbidities as a function of gender evidenced a significantly higher incidence of BPD and LOS in male infants, while we did not detect differences in the other comorbidities analyzed. Other studies in a Canadian population have also reported a higher risk of respiratory disorders, BPD and RDS in premature male infants ${ }^{9}$. Similar findings have been recently reported in a Japanese population of VLBW premature infants. However, this study did not analyze possible confounding factors, such as gestational age or birth weight, which are known to influence respiratory disorders in preterm infants ${ }^{11}$. In our study, adjusting for these possible confounding variables, the higher incidence of BPD in male infants remained significant. Therefore, we conclude that male gender is a risk factor for BDP in VLBW preterm infants.

Sepsis is a serious complication among preterm infants hospitalized in NICUs, particularly VLBW, increasing the risk of mortality. It has been reported that the risk of LOS is inversely related to birth weight and 
gestational age $\mathrm{e}^{19}$. To the best of our knowledge, there are no reports specifically evaluating the influence of gender on sepsis. Our study demonstrates a higher incidence of LOS during admission in male infants compared to females, and this difference remained statistically significant when adjusting by gestational age and birth weight. Therefore, we conclude that male gender is a risk factor for LOS in VLBW premature infants.

During intrauterine life, the fetus experiences the fastest growth between 22 and 40 weeks of gestation, increasing its weight over six-fold. Preterm infants who are born during this rapid growth phase are at a disadvantage. Therefore, growth during admission should be monitored to ensure optimum accretion, since it will influence neonatal outcome ${ }^{20}$. It is known that male fetuses grow faster than females, and these differences are observed from early gestation onward ${ }^{14}$. We evaluated the influence of gender on body anthropometric parameters and growth during admission. As previously reported, male infants exhibited a larger body weight and head circumference compared to females ${ }^{20}$. However, during hospitalization, there was no difference between the genders in any of the evaluated growth parameters. The lack of differences in growth rate might be related to the fact that all the infants were from a single NICU, where nutrition is optimized and individualized to obtain optimal fetal accretion.

EUGR is a serious problem which is closely related to growth and neurodevelopment outcomes. Furthermore, EUGR has also been linked to later alterations in cardiometabolic profiles. Therefore, the importance of nutrition and growth during the perinatal period must be emphasized. It is not known whether gender influences EUGR and if nutrition should be adjusted according to gender. A higher incidence of EUGR was found in male infants adequate for gestational age. However, this difference was not evident after adjusting for gestational age and birth weight, suggesting that gender is not a main factor in the development of EUGR.

Several mechanisms have been proposed to explain the higher incidence of prematurity in the male gender. The larger body weight of boys compared to females might increase the probability of preterm labor. A second possible explanation is the influence of the placenta, which is produced by the fetus, and thus carries the same biological gender ${ }^{21}$. Differences in the growth rate, structure, and function of the placenta between genders are well known, related to the differences in sexual hormones in early pregnancy. This has also been demonstrated in pregnancies complicated by fetal growth restriction, pre-eclampsia, and preterm birth ${ }^{22}$.
Infection and inflammation are also possible risk factors for preterm delivery. This is suggested by in vitro studies in placental or chorion trophoblast cells. These studies evidence that cells derived from male placenta respond to a stressor with a more pro-inflammatory cytokine pattern, compared to female-derived cells ${ }^{23}$. Gender differences in the placenta might also explain the higher incidence of LOS observed in male VLPW preterm infants. There is also evidence of gender-dependent differences in the transcription profile of the human placenta. In particular, the female placenta exhibits an upregulation of immune system-related genes $^{24}$, which has been proposed to equip females to respond better to potential infections ${ }^{21}$.

\section{Conclusion}

The main finding of the present study is that the male gender is associated with a higher incidence of BPD and LOS, two relevant pathologies related to prematurity in VLBW neonates. Furthermore, the worse outcome in male infants remained significant after adjusting for birth weight and gestational age as confounding variables. To the best of our knowledge, this is the first report in a cohort of VLBW infants showing the influence of gender on the incidence of comorbidities during the hospitalization period. The present study was obtained in a very homogeneous population of VLBW premature infants from a single NICU. It would be interesting to confirm the influence of gender on neonatal outcome in a larger population of preterm infants, including more than VLBW premature neonates. This information would be valuable in the consideration of specific gender-dependent care at NICUs.

\section{Financing}

PN I+D+I [FEM2015-63631-R] y ISCIII [PI16/00606].

\section{Conflicts of interest}

The authors declare no conflicts of interest regarding the publication of this article.

\section{Ethical disclosures}

Protection of human and animal subjects. The authors declare that the procedures followed were in accordance with the regulations of the relevant clinical research ethics committee and with those of the Code 


\section{of Ethics of the World Medical Association (Declaration of Helsinki).}

Confidentiality of data. The authors declare that they have followed the protocols of their work center on the publication of patient data.

Right to privacy and informed consent. The authors have obtained the written informed consent of the patients or subjects mentioned in the article. The corresponding author is in possession of this document.

\section{References}

1. Blencowe $\mathrm{H}$, Cousens $\mathrm{S}$, Chou D, et al. Born too soon: the global epidemiology of 15 million preterm births. Reprod Health. 2013;10 Suppl 1:S2.

2. Maitre NL, Ballard RA, Ellenberg JH, et al. Respiratory consequences of prematurity: evolution of a diagnosis and development of a comprehensive approach. J Perinatol. 2015;35:313-21.

3. Shaw AG, Sim K, Randell P, et al. Late-onset bloodstream infection and perturbed maturation of the gastrointestinal microbiota in premature infants. PLoS One. 2015;10:e0132923.

4. Shannon K, Mahmud Z, Asfia A, Ali M. The social and environmental factors underlying maternal malnutrition in rural Bangladesh: implications for reproductive health and nutrition programs. Health Care Women Int 2008;29:826-40.

5. Gotsch F, Gotsch F, Romero R, et al. The preterm parturition syndrome and its implications for understanding the biology, risk assessment, diagnosis, treatment and prevention of preterm birth. J Matern Fetal Neonatal Med. 2009;22 Suppl 2:5-23.

6. Zeitlin J, Saurel-Cubizolles MJ, De Mouzon J, et al. Fetal sex and preterm birth: Are males at greater risk? Hum Reprod. 2002;17:2762-8.

7. Xu H, Dai Q, Xu Y, et al. Time trends and risk factor associated with premature birth and infants deaths due to prematurity in Hubei province, China from 2001 to 2012. BMC Pregnancy Childbirth. 2015;15:329.

8. Peelen MJ, Kazemier BM, Ravelli AC, et al. Impact of fetal gender on the risk of preterm birth, a national cohort study. Acta Obstet Gynecol Scand. 2016;95:1034-41.
9. Ito M, Tamura M, Namba F; Neonatal Research Network of Japan. Role of sex in morbidity and mortality of very premature neonates. Pediatr Int 2017;59:898-905.

10. Kent AL, Wright IM, Abdel-Latif ME; New South Wales and Australian Capital Territory Neonatal Intensive Care Units Audit Group. Mortality and adverse neurologic outcomes are greater in preterm male infants. Pediatrics. 2012;129:124-31.

11. Ong KK, Ahmed ML, Emmett PM, Preece MA, Dunger DB. Association between postnatal catch-up growth and obesity in childhood: prospective cohort study. BMJ. 2000;320:967-71.

12. The CRIB (clinical risk index for babies) Score: A Tool for Assessing Initial Neonatal Risk and Comparing Performance of Neonatal Intensive Care Units. The international neonatal network. Lancet. 1993;342:193-8.

13. Luna MS, Hernando JM, Mussons FB, et al. Displasia broncopulmonar: definiciones y clasificación. Ann Pediatr. 2013;79:1-6.

14. Bell MJ, Ternberg JL, Feigin RD, et al. Neonatal necrotizing enterocolitis. Therapeutic decisions based upon clinical staging. Ann Surg. 1978; 187:1-7.

15. du Plessis AJ, Newburger J, Jonas RA, et al. Cerebral oxygen supply and utilization during infant cardiac surgery. Ann Neurol. 1995;37:488-97.

16. International Committee for the Classification of Retinopathy of Prematurity. The international classification of retinopathy of prematurity revisited. Arch Ophthalmol. 2005;123:991-9.

17. Patel AL, Engstrom JL, Meier PP, Kimura RE. Accuracy of methods for calculating postnatal growth velocity for extremely low birth weight infants. Pediatrics. 2005;116:1466-73.

18. Patel AL, Engstrom JL, Meier PP, Jegier BJ, Kimura RE. Calculating postnatal growth velocity in very low birth weight (VLBW) premature infants. J Perinatol. 2009;29:618-22.

19. Binet ME, Bujold $E$, Lefebvre $F$, et al. Role of gender in morbidity and mortality of extremely premature neonates. Am J Perinatol. 2012; 29:159-66.

20. Vatten LJ, Skjaerven R. Offspring sex and pregnancy outcome by length of gestation. Early Hum Dev. 2004;76:47-54

21. Di Renzo GC, Rosati A, Sarti RD, Cruciani L, Cutuli AM. Does fetal sex affect pregnancy outcome? Gend Med. 2007;4:19-30.

22. Melamed N, Yogev $\mathrm{Y}$, Glezerman M. Fetal gender and pregnancy outcome. J Matern Fetal Neonatal Med. 2010;23:338-44.

23. Tan $\mathrm{H}$, Wen SW, Walker $M$, et al. The association between fetal sex and preterm birth in twin pregnancies. Obstet Gynecol. 2004;103:327-32.

24. Stevenson DK, Verter J, Fanaroff AA, et al. Sex differences in outcomes of very low birthweight infants: the newborn male disadvantage. Arch Dis Child Fetal Neonatal Ed. 2000;83:F182-5. 